\title{
Assessment by general practitioners of suitability of thrombolysis in patients with suspected acute myocardial infarction
}

\author{
John D Gemmill, William K Lifson, Alan P Rae, W Stewart Hillis, Francis G Dunn
}

\begin{abstract}
Objective-To assess the clinical ability of general practitioners to decide to give thrombolytic therapy to patients with suspected myocardial infarction and to assess the contribution of the electrocardiograph (ECG) to this decision-making process.
\end{abstract}

Setting-7 practices on the North side of Glasgow and the coronary care unit of Stobhill General Hospital.

Subjects -137 patients presenting with chest pain who required direct admission to the coronary care unit.

Main outcome measures-Agreement between the general practitioner's clinical decision to give thrombolytic therapy with or without reference to the ECG and the prescription of thrombolytic therapy in the coronary care unit.

Results-The predictive accuracy of the general practitioner's assessment of the necessity for thrombolytic therapy was $71 \cdot 5 \%$. The ECG had no impact on the accuracy of this decision and there were problems with the recording and interpretation of the ECG. Clinical decision making was altered in six cases by the ECG-wrongly in four.

Conclusion-The diagnostic accuracy among general practitioners would result in some patients who did not have acute myocardial infarction being given thrombolytic therapy. In this study the ECG did not contribute towards diagnostic accuracy. Substantial improvement in both the recording and interpretation of ECGs is needed before thrombolytic agents can be routinely prescribed at home.

(Br Heart f 1993;70:503-506)

The time from onset of symptoms to the administration of thrombolytic therapy in acute myocardial infarction is a major influence on the derived benefits. ${ }^{12}$ Streamlining admission policies reduces unnecessary delay, ${ }^{3}$ and this delay could be further reduced by prehospital administration of thrombolytic agents, but the accuracy of diagnosis must be maintained to allow a satisfactory risk:benefit ratio. The administration of thrombolytic therapy in some studies has been based on a clinical assessment alone, ${ }^{4}$ whereas in other studies electrocardiographic (ECG) criteria have been used. ${ }^{5}$ This study was designed to assess the ability of general practitioners to decide appropriately on the prescription of thrombolytic therapy both on clinical grounds and with the addition of information provided by the ECG.

\section{Patients and methods}

We studied 139 patients assessed from August 1988 to August 1990. Two of these patients were excluded because the protocol was not observed. All the patients had summoned medical help because of persisting chest pain and in each case the general practitioner considered that direct admission to the coronary care unit was appropriate. This direct admission policy has been described before. ${ }^{3}$ It includes all patients thought by their attending general practitioner to have a suspected acute myocardial infarction. Those patients in whom the diagnosis of ischaemic chest pain was thought to be unlikely but who required further evaluation in hospital were referred to the accident and emergency department where they were assessed by the medical registrar. They are not included in this study. The patients were referred by 37 general practitioners in seven practices in the Stobhill catchment zone, and all the practices provided their own 24 hour clinical cover.

Before the start of the study, the general practitioners were familiarised with the standard indications and contraindications for thrombolytic therapy, and were instructed that those patients with electrocardiographic ST segment elevation of at least $1 \mathrm{~mm}$ in two standard leads or $2 \mathrm{~mm}$ in two precordial leads should receive thrombolytic therapy. Chest pain that the general practitioner thought merited admission to the coronary care unit was the sole criterion for entry into the study. ${ }^{3}$

The general practitioners were provided and familiarised with an ECG machine (Siemens Cardiostat 2) for each practice. In this study we specifically chose not to provide teaching sessions on ECG interpretation for the general practitioners, so that we could assess their current level of knowledge.

The general practitioners were asked whether on the basis of clinical evaluation they would give thrombolytic therapy and if not whether the reason was diagnostic uncertainty or contraindications. Half the patients were randomised to have an ECG in addition, and the same series of questions was asked after the recording and interpretation of the ECG. 
The hospital policy for administration of thrombolytic therapy was based on the history plus ECG criteria of at least $1 \mathrm{~mm} \mathrm{ST}$ elevation in two standard leads or $2 \mathrm{~mm} \mathrm{ST}$ elevation in two precordial leads in the absence of the accepted contraindications.

When they arrived at the coronary care unit the patients were reassessed by the attending medical registrar/senior house officer. The decision to give thrombolytic therapy was made on clinical grounds in conjunction with the ECG recorded at home and a second recorded on arrival in the coronary care unit, as is our standard practice. The reliability of the general practitioner's assessment, both on clinical grounds and with the electrocardiograph, was compared with the ultimate decision to give thrombolytic therapy, and the predictive accuracy was calculated. The ECGs recorded at home were subsequently reviewed by one of us.

\section{Results}

Fifty two of 137 patients had a diagnosis of acute myocardial infarction at the time of discharge from the coronary care unit and 26 of them had been given thrombolytic therapy (fig 1). None of the patients in this series who did not have a confirmed diagnosis of myocardial infarction was given thrombolytic therapy. In the 85 patients with a discharge diagnosis other than acute myocardial infarction, the diagnosis was unstable angina pectoris in 50, non-cardiac chest pain in 28, other in five, and two deaths before admission. Twenty five patients with acute myocardial infarction did not receive thrombolytic therapy, principally because they did not meet the ECG criteria (ST/T changes only, seven patients) or had contraindications (eight patients) (table). One patient received thrombolytic therapy late after admission because of recurrence of chest pain associated with ST elevation.

GENERAL PRACTITIONER ASSESSMENT COMPARED WITH TREATMENT RECEIVED IN THE CORONARY CARE UNIT

On the basis of clinical assessment alone, $49(35.8 \%)$ patients would have received thrombolytic therapy at home. Twenty seven patients had had a myocardial infarction and 18 of these ultimately received thrombolytic therapy in hospital (fig 2). Thrombolytic therapy was not given in the other patients because of previously undetected contraindications in five, death before admission in one, ST/T changes only in one, and an unspecified reason in two. The remaining 22 did not have a myocardial infarction and none was treated with thrombolytic therapy. Therefore on the basis of clinical assessment alone 27 patients would have been given thrombolytic therapy inappropriately: 22 did not have acute myocardial infarction and five had unrecognised contraindications.

Eighty eight $(64 \cdot 2 \%)$ patients would have been recommended by the general practitioners not to receive thrombolytic therapy. 25 of these had an acute myocardial infarction and 63 did not. Eight of these 88 patients ultimately received thrombolytic therapy and most of the 17 other patients with myocardial infarction did not receive thrombolytic therapy because of contraindications or ST/T changes only (table).

\section{ECG ANALYSIS}

Sixty nine patients were randomised to undergo ECG recording at home and this was successfully completed in $52(75 \%)$. The reasons for failure to record the ECG were documented in all 17. They were lack of time in six, machine malfunction in eight, machine not available in two, and one patient had a cardiac arrest before the recording was completed. In a further 11 patients the poor quality of the ECGs interfered with interpretation, rendering any interpretation impossible in three. The recording was satisfactory in $60 \%$.

In the group of 52 patients in whom ECG evaluation was complete the general practitioners would have treated 19 on clinical grounds only and not treated the remaining 33. After ECG evaluation, 19 patients would still have been treated. These were not the same 19 patients, seven of whom received thrombolytic therapy in the coronary care unit. The ECG reversed the treatment decision in six patients, wrongly in four.

The ECG recorded at home in all six patients for whom thrombolytic therapy was not recommended, but who were subsequently treated with thrombolytic therapy in the coronary care unit, showed diagnostic

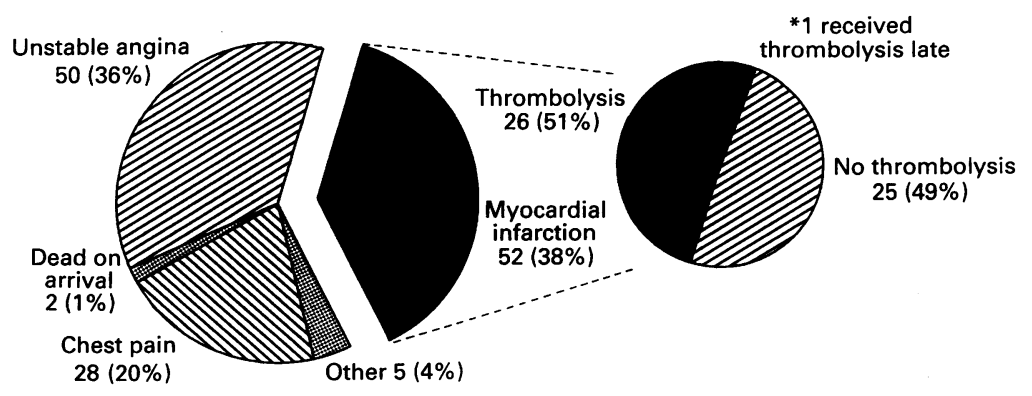

Figure 1 Discharge diagnosis in all patients and proportion of patients with acute myocardial infarction receiving thrombolytic therapy.
Reasons why patients with myocardial infarction did not receive thrombolytic therapy (25 patients)

\begin{tabular}{ll}
\hline Reason & Number (\%) \\
\hline Contraindications & $8(32)$ \\
Non-Q wave MI (ST/T changes only) & $7(28)$ \\
Unspecified reason & $7(28)$ \\
Patient had cardiac arrest & $1(4)$ \\
$\begin{array}{l}\text { Spontaneous resolution } \\
\text { of ECG changes }\end{array}$ & $1(4)$ \\
Left bundle branch block pattern & $1(4)$ \\
\hline
\end{tabular}

MI, myocardial infarction; ECG, electrocardiogram. 

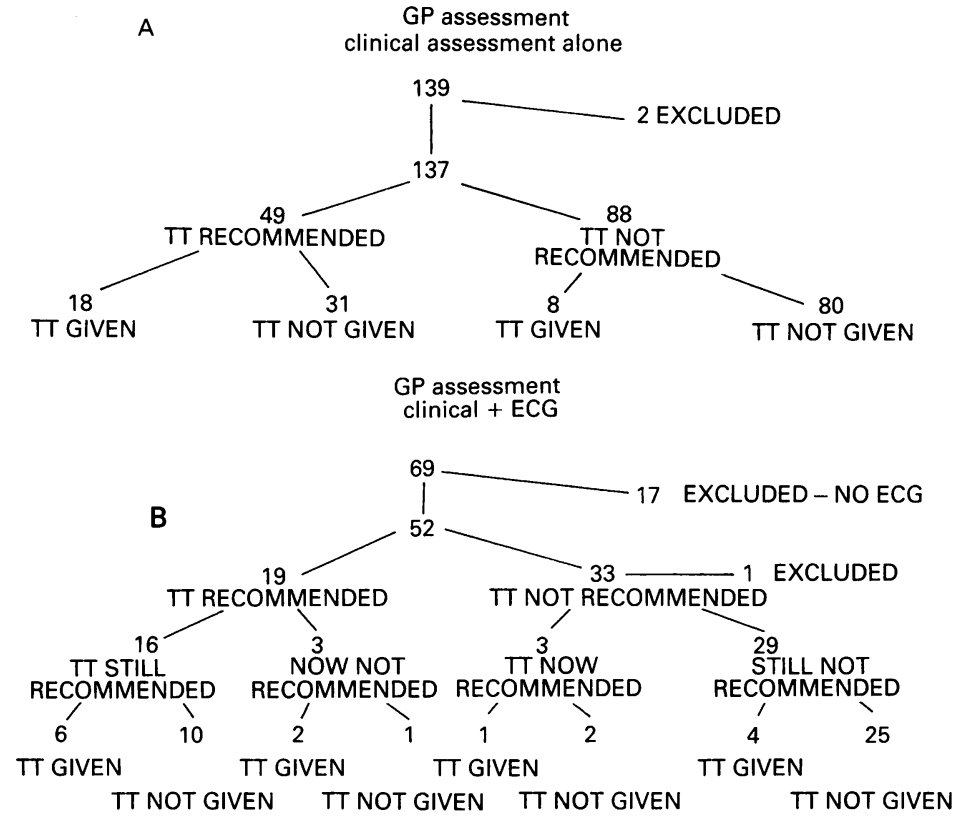

Figure 2 Flow diagram showing treatment recommended by general practitioner compared with prescription of thrombolytic therapy (TT) in the coronary care unit. (A) Based on clinical assessment alone. (B) Based on clinical assessment and ECG. unrecognised ST segment elevation. In the three patients in whom the clinical decision to give thrombolytic therapy was reversed by the ECG, there was ST elevation on the ECG recorded at home in two patients and $T$ wave changes in one. In the three patients in whom the decision not to give thrombolytic therapy was reversed after ECG analysis, ST elevation was seen in one patient, but unfortunately the remaining two ECGs recorded at home were not available for analysis in the hospital.

Where the recording of the ECG did not influence the general practitioner's clinical decision to give thrombolysis (16 patients), the ECG was normal in two, showed ST elevation in 10, ST depression in one, and non-specific ST/T changes in the remainder. Where the recording of the ECG did not influence the clinical decision not to treat with thrombolytic therapy (29 patients) the ECG was normal in 12; showed old ischaemic changes in two, left ventricular hypertrophy in three, non-specific ST/T changes in two, and left bundle branch block pattern in one; was uninterpretable or unavailable in three; and showed ST elevation in six. The reason given for not advising thrombolytic therapy in the five patients with ST elevation was diagnostic uncertainty in four and contraindications in one.

The predictive accuracy in the group not undergoing ECG recording was $71.5 \%$. In the group undergoing ECG the predictive accuracy was $68.6 \%$ on clinical grounds alone. This fell to $64 \cdot 7 \%$ once the information from the completed ECG was incorporated into the decision-making process.

\section{Discussion}

The benefits of thrombolytic therapy are greatest when treatment is given early. ${ }^{14}$ The different components in the delay to administration of thrombolytic therapy have been documented $^{6}$ and the hospital delay can be reduced by a direct admission policy. ${ }^{37}$ One logical approach to reducing the prehospital delay is for thrombolytic therapy to be administered in the patient's home by the general practitioner. However, any such treatment requires risk:benefit ratio analysis.

Thrombolytic therapy is not without risk, especially when administered to patients with a diagnosis other than myocardial infarction ${ }^{89}$ or to patients with any of the recognised contraindications. The risks of thrombolytic therapy within hospital are small, however, when administered to a selected population, ${ }^{10}$ and early fears of reperfusion arrhythmia or injury were shown to be unfounded in clinical practice. ${ }^{1}$

Studies of thrombolytic therapy administered in the community have been performed both in the United Kingdom and elsewhere. Out-of-hospital thrombolytic therapy is feasible, reasonably safe, shortens the time to treatment by 34-68 minute s $^{11-13}$ in an urban setting, and further reduces mortality compared with hospital administration, at least in a rural setting where the median time saved was 130 minutes. ${ }^{14} \mathrm{~A}$ previous study by our group showed a mean transit time from home to hospital of 35 minutes, ${ }^{3}$ which equates with the time saved by domiciliary administration of thrombolytic therapy in this study.

When general practitioners based their assessment of the need for thrombolytic therapy on clinical grounds alone the predictive accuracy for appropriate use of these drugs was low, which accords with a recently published study ${ }^{15}$ in which there was no systematic analysis of the importance of the ECG in decision-making.

Current opinion, which indicates that thrombolytic therapy is best administered to those patients with definite ECG evidence of acute myocardial infarction, ${ }^{16}$ is largely based on concerns about misdiagnosis and the suggestion that those patients without changes diagnostic of acute myocardial infarction on the ECG have a better prognosis and derive less benefit from thrombolytic therapy than those with ST elevation. ${ }^{41718}$ The treatment rules used in our study were those current at the time of the start of the study, and they reflect a high threshold for administration of thrombolytic therapy. In particular, our adherence to ECG criteria of ST elevation excluded from therapy those patients with a true posterior site of infarction or without ST elevation (non- $Q$ wave myocardial infarction) or with a left bundle branch block pattern.

This makes the comparison of the general practitioner's decision-making with that within the coronary care unit more rigorous, but this is appropriate if ECG criteria are a prerequisite for the prescription of thrombolytic therapy. If administration of thrombolytic therapy at home were based on the 
clinical diagnosis of myocardial infarction many patients would be treated inappropriately.

In our study ECGs were successfully recorded at home in only $60 \%$ of patients and the poor quality of the recording interfered with the interpretation of $20 \%$ of the traces. The two most obvious problems identified were $50 \mathrm{~Hz}$ mains interference, presumably from domestic appliances, and patient movement and tremor artefact, reflecting the patient's distress. Even when the trace was satisfactory the clinical decision was altered on only șix occasions. Although the ECG was of assistance in assessing whether the pain was ischaemic it did little to influence the accuracy of prediction of which patients would be treated with thrombolysis in the coronary care unit. In fact on four of the six occasions the decision was changed in an inappropriate direction. In those six patients in whom thrombolytic therapy was not recommended at home, but who subsequently received therapy in the coronary care unit, the ECG recorded at home showed diagnostic changes of acute myocardial infarction in all six, suggesting that the interpretation of ECGs at home is difficult. This also suggests that the development of ST changes during the patients' transfer to hospital did not explain the discrepancy.

In our study the ECG was of little benefit to the attending general practitioner. Some of the problems encountered may be partly remediable with increased familiarity with the ECG machines and interpretation of the recordings or with the use of self-interpreting or trans-telephonic devices.

Others have drawn attention to the low usage of ECGs in general practice. ${ }^{1920}$ If an ECG analysed in the patient's home is going to be a prerequisite for the treatment of acute myocardial infarction, general practitioners will need additional training in both the recording and interpretation of the ECG and also encouragement to obtain the necessary equipment. ${ }^{21}$

The British Heart Foundation Working Group recommends that until there is adequate assessment of the safety and improved efficacy of the administration of thrombolytic therapy at home, the emphasis should be on streamlining the transport of the patient to a hospital that is experienced with the use of thrombolytic therapy where continuous monitoring by experienced staff is available. ${ }^{22}$ This view is endorsed by others, ${ }^{15}{ }^{23}$ and by our experience in this study.

We thank the 37 general practitioners who participated in this study

1 Gruppo Italiano Per Lo Studio Dela Streptochinasi nell' Infarto Miocardico (GISSI). Effectiveness of intravenous thrombolytic treatment in acute myocardial infarction. Lancet 1986;i:397-401.

2 Simoons ML, Serruys PW, van der Brand M, et al. Early thrombolysis in acute myocardial infarction: limitation of infarct size and improved survival. $\mathcal{F} \mathrm{Am}$ Coll Cardiol 1986;7:717-28.

3 Burns JMA, Hogg KJ, Rae AP, Hillis WS, Dunn FG. Impact of a policy of direct admission to a coronary care unit on use of thrombolytic therapy. Br Heart 71989 61:322-5.

4 ISIS-2 Collaborative Group. Randomised trial of intravenous streptokinase, oral aspirin, both, or neither among 17187 cases of suspected acute myocardial infarction: ISIS-2. Lancet 1988;ii:349-60.

5 Wilcox RG. Thrombolysis with tissue plasminogen activator in suspected acute myocardial infarction. The vator in suspected acute myocardial
ASSET Study. Chest 1989;95:270S-5S

6 Pell ACH, Miller HC. Delays in admission of patients with acute myocardial infarction to coronary care: Implications for thrombolysis. Health Bull (Edinb) 1990;48:225-31.

7 Pell ACH, Miller HC, Robertson CE, Fox KAA. Effect of "fast track" admission for acute myocardial infarction on delay to thrombolysis. $\mathrm{BrMed} \mathcal{F} 1992 ; 304: 83-7$.

8 Butler J, Davies AH, Westaby S. Streptokinase in acute aortic dissection. Br Med ₹ 1990;300:517-9.

9 Curzen NP, Clarke B, Gray HH. Intravenous thrombolysis for suspected myocardial infarction: a cautionary note. $\mathrm{BrMed} \mathcal{F}$ 1990;300:513-4.

10 Petch MC. Dangers of thrombolysis. $\mathrm{Br}$ Med $\mathcal{F} 1990$; 300:483.

11 Roth A, Brabash CI, Hod H. Should rt-PA be administrated by the mobile intensive care unit team? [abstr] Circulation 1988;78(suppl): 189.

12 Castaigne $\mathrm{AD}$, Herve $\mathrm{C}$, Duval-Moulin $\mathrm{AM}$, et al. Prehospital use of APSAC: results of a placebo-controlled study. Am $\mathcal{F}$ Cardiol 1989;64(suppl A):30-3.

13 McNeill A, Cunningham S, Flannery D, et al. Preadmission recombinant tissue plasminogen activator [abstr]. Eur Heart $\mathcal{f} 1988 ; 9$ (suppl 1):214.

14 GREAT Group. Feasability, safety, and efficacy of domiciliary thrombolysis by general practitioners: Grampian region early anistreplase trial. $\mathrm{Br} \mathrm{Med} \mathcal{f} 1992 ; 305$ 548-53.

15 Rule S, Brooksby P, Sanderson J. Use of thrombolysis for acute myocardial infarction by general practitioners. Postgrad Med F 1993;69:190-3.

16 Timmis AD. Early diagnosis of acute myocardial infarction. Electrocardiography is still best. $\mathrm{Br} \mathrm{Med} \mathcal{F} 1991$; 301:941-2.

17 Wilcox RG, von der Lippe G, Olsson CG, Jensen G Skene AM, Hampton JR. Trial of intravenous tissue plasminogen activator for mortality reduction in acute myocardial infarction. Anglo-Scandinavian study early thrombolysis. Lancet 1988;ii:525-30.

18 Muller DWN, Topol EJ. Selection of patients with acute myocardial infarction for thrombolytic therapy. Ann Intern Med 1990;113:949-60.

19 Bradley N, Watkins S. Survey of equipment in general practice. Br Med F 1989;299:435-6.

20 Colquhoun MC. General practitioners' use of electrocardiography: relevance to early thrombolytic treatment. cardiography: relevance to early thrombolytic treatment.

21 Davies A. Electrocardiographs in general practice. $\mathrm{Br} \mathrm{Med}$ f 1989;299:408-9.

22 Report of a British Heart Foundation Working Group. Role of the general practitioner in managing patients with myocardial infarction: impact of thrombolytic treatment. Br Med f 1989;299:555-7.

23 Fox KAA. Thrombolysis and the general practitioner. Practicable only under certain circumstances. $\mathrm{Br} M e d \mathcal{F}$ 1990;300:867-8. 